\title{
Ensemble quantum-information processing by NMR: Spatially averaged logical labeling technique for creating pseudopure states
}

\author{
T. S. Mahesh ${ }^{1}$ and Anil Kumar ${ }^{1,2, *}$ \\ ${ }^{1}$ Department of Physics, Indian Institute of Science, Bangalore 560012, India \\ ${ }^{2}$ Sophisticated Instruments Facility, Indian Institute of Science, Bangalore 560012, India
}

\begin{abstract}
A method for creating pseudopure states in NMR is described that combines spatial averaging and logical labeling techniques. This method uses one labeling qubit and any desired pseudopure state of any number of qubits can be created in the subsystem manifolds of the labeling qubit. The method uses a nonselective $\pi / 2$ radio frequency pulse on all the nonlabeling qubits to equalize the populations of all the states within each subsystem, followed by a single transition-selective $\pi$ pulse on one of the transitions of the labeling qubit. The transverse magnetization created during the process is destroyed by a magnetic field gradient pulse, yielding a subsystem pseudopure state. The method is demonstrated experimentally on a system of three qubits.
\end{abstract}

\section{INTRODUCTION}

Quantum-information processing often needs to begin from a pure state. Examples are Shor's factorizing algorithm [1] and Grover's search algorithm [2]. In the case of nuclear magnetic resonance (NMR) spectroscopy, the creation of a pure state requires unrealistic experimental conditions like near-zero temperatures or infinitely high magnetic fields. However, one can mimic pure states by creating pseudopure states or effective pure states. Several methods have been proposed and implemented for creating pseudopure states in NMR [3-20]. These include (i) spatial averaging [3,6], (ii) temporal averaging [5], and (iii) logical labeling [4,7,12]. Spatial averaging is based on step by step creation of basic product matrices using radio frequency (rf) pulses and magnetic field gradients, which finally lead to a pseudopure state. Temporal averaging is based on classical randomization and involves multiple experiments to achieve an effective pure state. The method does not use gradients and hence involves no loss of magnetization. Logical labeling is based on the fact that, by performing suitable permutation operations on the total spin system, subsystems in the density matrix can be reduced to pseudopure states [11]. This method requires an ancillary spin for labeling. Many of the existing methods are limited by the fact that the number of required rf pulses and scalar coupling evolution periods increases rapidly with increase in the number of qubits, which results in highly complicated pulse sequences and severe errors due to pulse imperfections. For example, while the spatial averaging technique for creating a pseudopure state in a two-qubit system needs three rf and two gradient pulses and two $J$-evolution periods, for a three-qubit system it needs eight $\mathrm{rf}$ and four gradient pulses and three $J$-evolution periods [6]. The logical labeling technique, on the other hand, produces two two-qubit subsystem pseudopure states (e.g., 00 and 11)

\footnotetext{
*Author to whom correspondence should be addressed. Email address: anilnmr@physics.iisc.ernet.in
}

in a three-qubit system, utilizing two transition-selective pulses [12]. For a four-qubit system, this method would still produce two-qubit subsystem pseudopure states (four of them: e.g., 00,11,00,11) utilizing four transition-selective pulses. In general, in the logical labeling method, the subsystem dimension is limited by the number of equally populated states, which for an $n$-qubit system is $C_{n / 2}^{n}$, yielding an $m$-qubit pseudopure state where $m=\log _{2}\left(1+C_{n / 2}^{n}\right)$ [10]. For heteronuclear systems, the situation is more complex and the ratio $m / n$ is generally smaller. In the temporal averaging technique for creating pseudopure states, the number of experiments increases exponentially to $\left(2^{n}-1\right)$ [5].

Here we propose a method for creating subsystem pseudopure states that combines both spatial averaging and logical labeling techniques. The method is based on choosing an extra spin as a labeling spin, which divides the spin system into two subsystems, equalizing the populations within each subsystem by applying a $\pi / 2 \mathrm{rf}$ pulse on all the nonlabeling spins, selectively inverting populations of one particular transition of the labeling spin, and then applying a magnetic field gradient to destroy all the coherences created during the process. The resulting longitudinal state yields the desired pseudopure state in the subsystem manifold (Fig. 1). This method needs only one $\pi / 2$ pulse on the nonlabeling spins, one transition-selective $\pi$ pulse on the labeling spin, and one gradient pulse to create any of the $2^{n}$ pseudopure states in any $(n+1)$-qubit system.

\section{THEORY}

Consider an $(n+1)$-spin system $I_{0}, I_{1}, I_{2}, \ldots, I_{n}$, in which spin $I_{0}$ is chosen as the labeling qubit. The density matrix of the spin system in thermal equilibrium can be written as

$$
\rho=\sum_{s=1}^{2^{n+1}} p_{s}|s\rangle\langle s|,
$$

where $p_{s}$ is the Boltzmann population for the state $|s\rangle$. The 

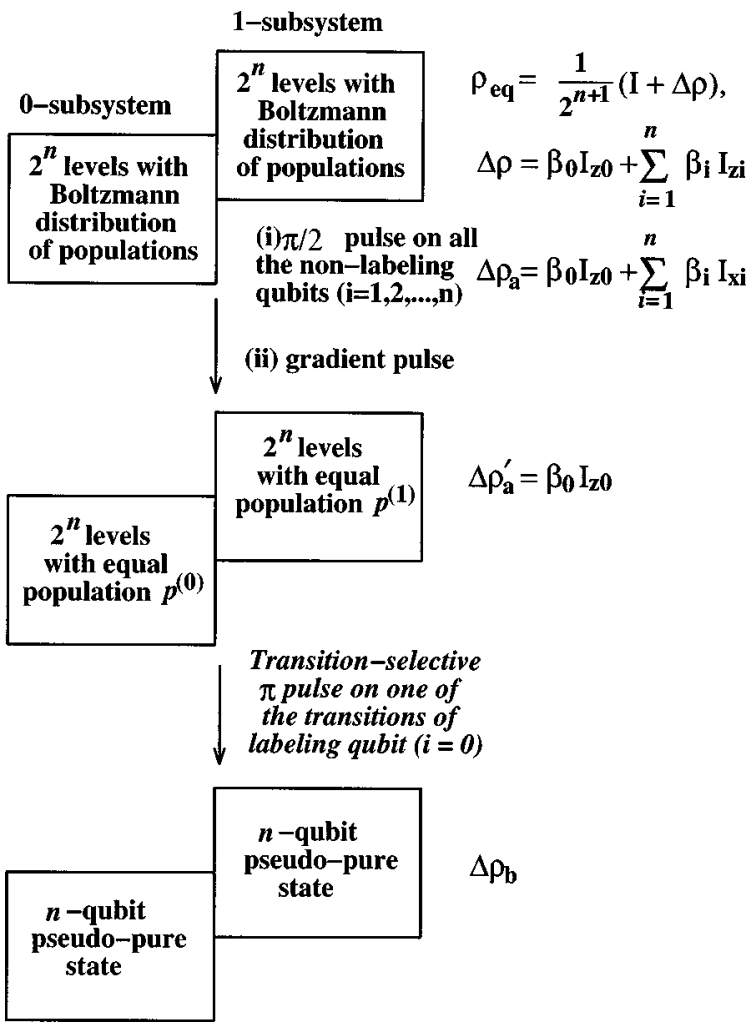

FIG. 1. Steps for creating pseudopure states by spatially averaged logical labeling technique (SALLT), which combines both logical labeling and spatial averaging techniques. The $2^{n+1}$ energy levels of the $(n+1)$-qubit system are divided into two subsystems: 0 subsystem and 1 subsystem, depending on the 0 and 1 states of the labeling qubit. The equilibrium density matrix $(\rho)$ and the deviation density matrices at various stages $\left(\Delta \rho, \Delta \rho_{a}, \Delta \rho_{a}^{\prime}\right.$, and $\left.\Delta \rho_{b}\right)$ are given in the right-hand side, and are discussed in the text. $I$ is the unit matrix and $\beta_{i}=\gamma_{i} \hbar H_{0} / k T$.

above expression can be rearranged into two parts corresponding to the two states $|0\rangle$ and $|1\rangle$ of the labeling qubit as

$$
\rho=\sum_{i=1}^{2^{n}} p_{i}^{(0)}|i\rangle\left\langle i\left|+\sum_{j=1}^{2^{n}} p_{j}^{(1)}\right| j\right\rangle\langle j|,
$$

where $|i\rangle$ and $|j\rangle$ are of the form $\left|0 i_{1} i_{2} \cdots i_{n}\right\rangle$ and $\left|1 j_{1} j_{2} \cdots j_{n}\right\rangle$, respectively. We call these two parts the 0 subsystem and 1 subsystem. The steps for creation of pseudopure states are shown in Fig. 1.

Starting from the thermal equilibrium, the first step is to equalize the populations of all the states within each subsystem. This can be achieved by a nonselective $\pi / 2 \mathrm{rf}$ pulse on all the $n$ nonlabeling spins [21]. All the off-diagonal terms in the density matrix created by the $\pi / 2$ pulse will be destroyed by a subsequent magnetic field gradient pulse, and hence off-diagonal elements will not be considered in the following. The density matrix can then be written as

$$
\rho_{a}=\sum_{i=1}^{2^{n}} p^{(0)}|i\rangle\left\langle i\left|+\sum_{j=1}^{2^{n}} p^{(1)}\right| j\right\rangle\langle j|,
$$

where $p^{(0)}$ and $p^{(1)}$ are the average populations of the 0 subsystem and 1 subsystem, respectively given by

$$
\begin{gathered}
p^{(0)}=\left(1 / 2^{n}\right) \sum_{i=1}^{2^{n}} p_{i}^{(0)} \\
\text { and } \quad p^{(1)}=\left(1 / 2^{n}\right) \sum_{j=1}^{2^{n}} p_{j}^{(1)} .
\end{gathered}
$$

Since the two subsystems are shifted by the Zeeman energy $\Delta E$ of the labeling spin (see Fig. 3 below),

$$
\frac{p^{(0)}-p^{(1)}}{p^{(0)}} \simeq \frac{\Delta E}{k T}=\frac{\gamma_{l} \hbar \mathrm{H}_{0}}{k T},
$$

where $\gamma_{l}$ is the gyromagnetic ratio of the labeling spin and $H_{0}$ is the magnetic field strength [22]. Any desired pseudopure state $\left|k_{1} k_{2} \cdots k_{n}\right\rangle$ of the subsystems can now be created by interchanging the populations of states $\left|0 k_{1} k_{2} \cdots k_{n}\right\rangle$ and $\left|1 k_{1} k_{2} \cdots k_{n}\right\rangle$ by a transition-selective $\pi$ pulse on the particular transition of the labeling spin. The density matrix is then given by

$$
\begin{aligned}
\rho_{b}= & {\left[\left(p^{(1)}-p^{(0)}\right)\left|k^{(0)}\right\rangle\left\langle k^{(0)}\left|+\sum_{i=1}^{2^{n}} p^{(0)}\right| i\right\rangle\langle i|\right] } \\
& +\left[\left(p^{(0)}-p^{(1)}\right)\left|k^{(1)}\right\rangle\left\langle k^{(1)}\left|+\sum_{j=1}^{2^{n}} p^{(1)}\right| j\right\rangle\langle j|\right],
\end{aligned}
$$

where $k^{(0)}$ and $k^{(1)}$ are, respectively, $\left|0 k_{1} k_{2} \cdots k_{n}\right\rangle$ and $\left|1 k_{1} k_{2} \cdots k_{n}\right\rangle$. The deviation density matrices $\Delta \rho_{b}$ for the two subsystems now correspond to pseudopure states and can be written as

$$
\begin{gathered}
\Delta \rho_{b}^{(0)}=\left(p^{(1)}-p^{(0)}\right)\left|k^{(0)}\right\rangle\left\langle k^{(0)}\right| \\
\text { and } \Delta \rho_{b}^{(1)}=\left(p^{(0)}-p^{(1)}\right)\left|k^{(1)}\right\rangle\left\langle k^{(1)}\right| .
\end{gathered}
$$

Any desired pseudopure state of subsystems can thus be created for any number of qubits.

The circuit for creating pseudopure states by this method is given in Fig. 2. It may be remarked that here the Hadamard gates have been utilized for equalizing the populations of all the states within each subsystem rather than for creating superpositions. This can also be achieved by pseudoHadamard gates [23], which can be implemented by applying a single $\pi / 2$ pulse on all nonlabeling qubits. The created superposition is destroyed by a gradient pulse (crusher). The special C-NOT gate corresponds to a labeling spin transitionselective $\pi$ pulse applied on a transition connecting the states that correspond to the desired pseudopure states.

\section{EXPERIMENT}

The pulse scheme for creating pseudopure states by this method is given in Fig. 3. The experiment is demonstrated on a system of three weakly coupled ${ }^{1} \mathrm{H}$ nuclear spins of 2,3-dibromo propionic acid dissolved in chloroform at $300 \mathrm{~K}$ 


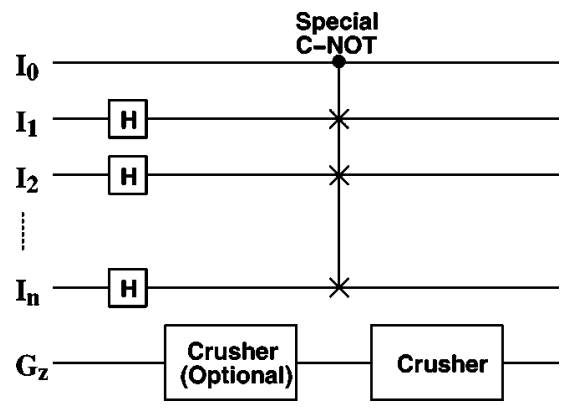

FIG. 2. Circuit for creating pseudopure states by SALLT method. Initially Hadamard gates are applied on all the nonlabeling qubits to equalize the populations within the subsystems. A special controlled-NOT (C-NOT) gate flips the state of the labeling spin only for a desired pseudopure state of the nonlabeling spins (a normal C-NOT gate leads to a $|111 \cdots 1\rangle$ pseudopure state, and a special C-NOT gate can lead to a desired pseudopure state). All the coherences generated are destroyed by a crusher.

in a Bruker DRX $500 \mathrm{MHz}$ spectrometer. The equilibrium spectrum of the sample is shown in Fig. 4(a). The three ${ }^{1} \mathrm{H}$ nuclear spins are labeled as $I_{0}, I_{1}$, and $I_{2}$, where $I_{0}$ is chosen as the labeling spin. Every transition of a spin between two states is labeled by the states of the other two spins.

In the beginning, a single $\pi / 2 \mathrm{rf}$ pulse on $I_{1}$ and $I_{2}$ spins is applied, which is followed by a transition-selective $\pi$ pulse on one of the transitions of the labeling qubit. The experiment is not sensitive to the angle imperfections of the $\pi$ pulse as long as its selectivity is good. However, maximum magnetization will be obtained when the pulse corresponds to $180^{\circ}$, since this creates maximum difference between the average background population and the population of the pseudopure state. A final magnetic field gradient pulse destroys all the off-diagonal elements created by the $\pi / 2$ pulse as well as by the nonideality of the $\pi$ pulse. The gradient pulse could also be applied immediately after the $\pi / 2$ pulse. In that case, however, another gradient pulse would be required at the end of the transition-selective $\pi$ pulse to remove the transverse magnetization created by the nonideality of the $\pi$ pulse. The pseudopure state thus created is measured by a nonselective small-angle rf pulse, such that the

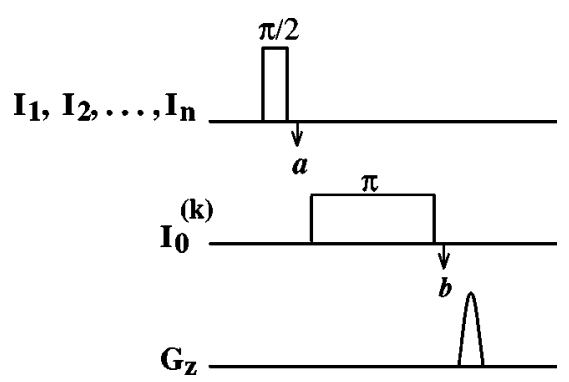

FIG. 3. Pulse sequence for creation of subsystem pseudopure states. The $\pi / 2$ pulse is applied on all the nonlabeling qubits $I_{1}, I_{2}, \ldots, I_{n}$ and the $\pi$ pulse is applied selectively on the $k$ th transition of the labeling qubit $I_{0}$. The pulse field gradient $\left(G_{z}\right)$ destroys all the transverse magnetization. The density matrices at instants $a$ and $b$ are discussed in the text.

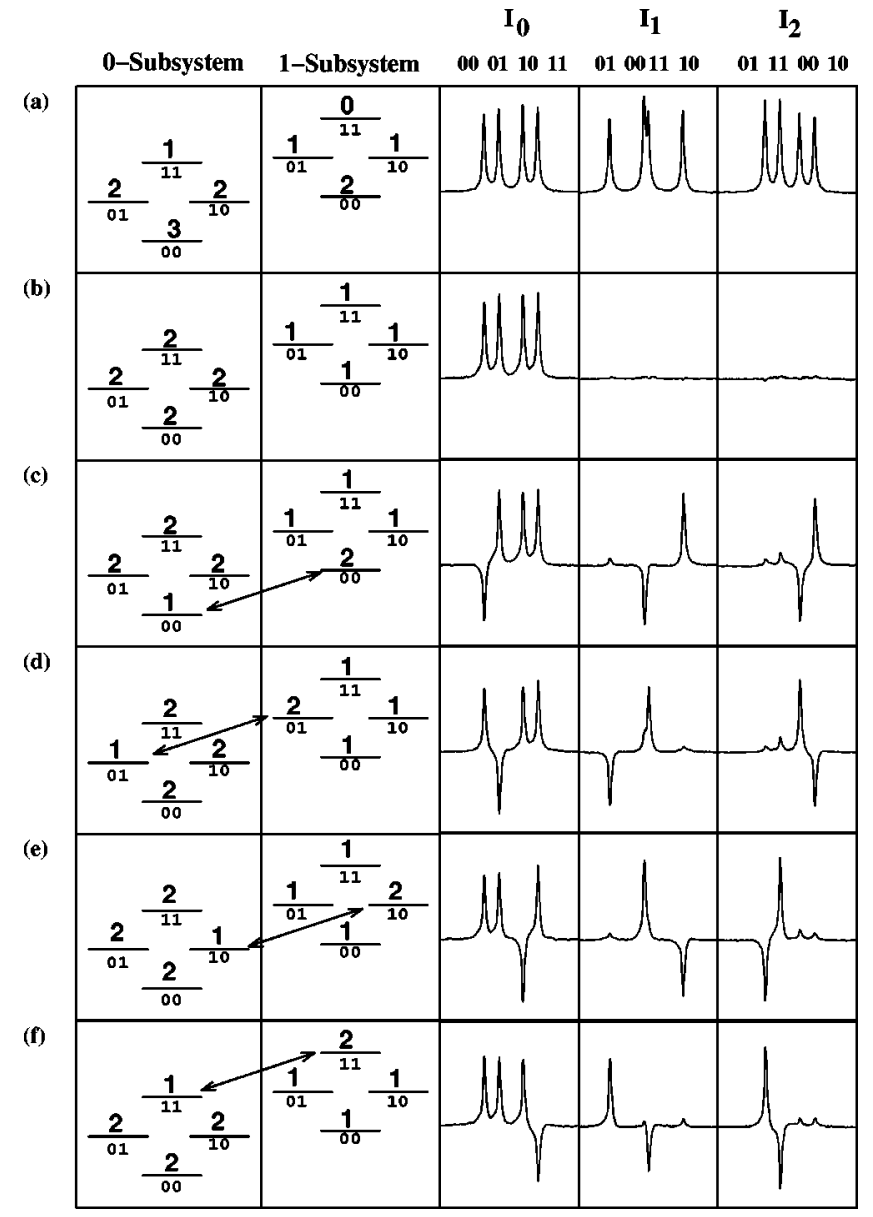

FIG. 4. The first column of the figure contains the energy-level diagrams of a homonuclear three-spin system with the numbers above and below the energy levels, respectively, indicating the relative population deviations and the states of the nonlabeling spins. The three spins correspond to three weakly coupled ${ }^{1} \mathrm{H}$ nuclei of 2,3-dibromo propionic acid dissolved in chloroform at $300 \mathrm{~K}$ in a Bruker DRX 500 spectrometer [12]. (a) Equilibrium populations and the corresponding spectra of the three spins. (b) The populations and the spectra obtained after applying a $\pi / 2$ pulse on spins $I_{1}$ and $I_{2}$, followed by a gradient pulse. (c), (d), (e), and (f) are the populations and corresponding spectra for the pseudopure states $|00\rangle,|01\rangle,|10\rangle$, and $|11\rangle$, respectively, obtained by applying selective $\pi$ pulses on transitions indicated by the arrows. All the spectra were obtained using a small-angle $\left(9^{\circ}\right)$ detection pulse of duration $0.86 \mu \mathrm{s}$. The $\pi / 2 \mathrm{rf}$ pulse on $I_{1}$ and $I_{2}$ was of Gaussian shape and $9 \mathrm{~ms}$ duration. The transition-selective $\pi$ pulse on one of the transitions (indicated by an arrow) $I_{0}$ was rectangular in shape and of $220 \mathrm{~ms}$ duration. A phase cycle $(x,-x)$ was employed for the transition-selective pulse to minimize the pulse imperfection effects. The magnetic field gradient pulse was of duration $2 \mathrm{~ms}$ and of strength $30 \mathrm{G} / \mathrm{cm}$.

intensity of a transition between two states is proportional to the difference between the populations in the two states [21]. The results are shown in Fig. 4, along with the energy-level diagrams showing relative population deviations (relative deviation from a uniform background population). Figure 4(b) shows the energy-level diagram with relative population deviations after applying a $\pi / 2$ pulse on the nonlabeling spins, 
and the corresponding spectrum obtained by using a smallangle detection pulse. Figures 4(c), (d), (e), and (f), respectively, correspond to the pseudopure states $|00\rangle,|01\rangle,|10\rangle$, and $|11\rangle$. The fidelity of the pseudopure states obtained in the present experiments is $(95 \pm 5) \%$. However, the fidelity may not remain so high as the number of qubits increases, due to selectivity problems, scaling effects, and decoherence effects [24].

It may be remarked that the labeling and nonlabeling qubits in the present case were ${ }^{1} \mathrm{H}$ spins. Therefore, the $\pi / 2$ pulse on nonlabeling qubits was a selective pulse of duration $9 \mathrm{~ms}$. However, if the labeling spin is chosen to be a heteronuclear spin, the $\pi / 2$ pulse on nonlabeling qubits will become very short (of the order of a few microseconds), further improving the performance of this method. Also, if the nonlabeling spins are not of the same gyromagnetic ratio, then more than one $\pi / 2$ pulse will be required to equalize the populations. Recently another method has been suggested for creating pseudopure states in the subsystems using an ancillary qubit [15]. However, this method requires scalar coupling evolution periods, which are not used in our method.

\section{CONCLUSIONS}

Significant progress has been achieved toward quantuminformation processing by NMR by the use of spin- and transition-selective pulses [12,13,17,25-28]. Further progress toward the implementation of quantum algorithms requires efficient preparation of pseudopure states. This paper demonstrates a method of creating pseudopure states in NMR using spin- and transition-selective pulses. The creation of pseudopure states of any number of qubits by this method is straightforward and requires the same pulse sequence without any increase in number of pulses or delays, yielding high fidelity subsystem pseudopure states. The scaling of this method, however, is the same as that of the spatial averaging method.

\section{ACKNOWLEDGMENTS}

Use of the $500 \mathrm{MHz}$ FTNMR spectrometer of the Sophisticated Instruments Facility (SIF), Indian Institute of Science, funded by the Department of Science and Technology, New Delhi is gratefully acknowledged. We also thank Professor K. V. Ramanathan and Dr. Arvind for discussions.
[1] P.W. Shor, SIAM J. Comput. 26, 1484 (1997).

[2] L.K. Grover, Phys. Rev. Lett. 79, 325 (1997).

[3] D.G. Cory, A.F. Fahmy, and T.F. Havel, Proc. Natl. Acad. Sci. U.S.A. 94, 1634 (1997).

[4] N. Gershenfeld and I.L. Chuang, Science 275, 350 (1997).

[5] E. Knill, I.L. Chuang, and R. Laflamme, Phys. Rev. A 57, 3348 (1998).

[6] D.G. Cory, M.D. Price, and T.F. Havel, Physica D 120, 82 (1998).

[7] I.L. Chuang, N. Gershenfeld, M.G. Kubinec, and D.W. Leung, Proc. R. Soc. London, Ser. A 454, 447 (1998).

[8] D.G. Cory, W. Maas, M. Price, E. Knill, R. Laflamme, W.H. Zurek, T.F. Havel, and S.S. Somaroo, Phys. Rev. Lett. 81, 2152 (1998).

[9] E. Knill, R. Laflamme, R. Martinez, and C.H. Tseng, Nature (London) 396, 52 (1998).

[10] L.M.K. Vandersypen, C.S. Yannoni, M.H. Sherwood, and I.L. Chuang, Phys. Rev. Lett. 83, 3085 (1999).

[11] M. A. Nielsen, and I. L. Chuang, Quantum Computation and Quantum Information (Cambridge University Press, Cambridge, 2000).

[12] Kavita Dorai, Arvind, and Anil Kumar, Phys. Rev. A 61, 042306 (2000).

[13] Kavita Dorai, T.S. Mahesh, Arvind, and Anil Kumar, Curr. Sci. 79, 1447 (2000).

[14] U. Sakaguchi, H. Ozawa, and T. Fukumi, Phys. Rev. A 61, 042313 (2000).
[15] Y. Sharf, T.F. Havel, and D.G. Cory, Phys. Rev. A 62, 052314 (2000).

[16] A.K. Khitrin and B.M. Fung, J. Chem. Phys. 112, 6963 (2000).

[17] Neeraj Sinha, T.S. Mahesh, K.V. Ramanathan, and Anil Kumar, J. Chem. Phys. 114, 4415 (2001).

[18] E. Knill, R. Laflamme, R. Martinez, and C.H. Tseng, Nature (London) 404, 368 (2000).

[19] B.M. Fung, Phys. Rev. A. 63, 022304 (2001).

[20] X. Peng, X. Zhu, X. Fang, M. Feng, K. Gao, and M. Liu, e-print quant-ph/0012038.

[21] R.R. Ernst, G. Bodenhausen, and A. Wokaun, Principles of Nuclear Magnetic Resonance in One and Two Dimensions (Oxford University Press, Oxford, 1987).

[22] A. Abragam, The Principles of Nuclear Magnetism (Oxford University Press, Oxford, 1961).

[23] J.A. Jones, R.H. Hansen, and M. Mosca, J. Magn. Reson. 138, 353 (1998).

[24] C.H. Tseng, S. Somaroo, Y. Sharf, E. Knill, R. Laflamme, T.F. Havel, and D.G. Cory, Phys. Rev. A 62, 032309 (2000).

[25] N. Linden, H. Barjat, and R. Freeman, Chem. Phys. Lett. 296, 61 (1998).

[26] T.S. Mahesh, Kavita Dorai, Arvind, and Anil Kumar, J. Magn. Reson. 148, 95 (2001).

[27] Kavita Dorai, Arvind, and Anil Kumar, Phys. Rev. A 63, 034101 (2001).

[28] Arvind, Kavita Dorai, and Anil Kumar, Pramana 56, L705 (2001), e-print quant-ph/9909067. 